\title{
ОЦЕНКА КАЛЕНДАРНЫХ ПЛАНОВ, ПРИМЕНЯЕМЫХ В ОПЕРАТИВНОМ И ТАКТИЧЕСКОМ УПРАВЛЕНИИ
}

\author{
П. М. Кузьмич1', Е. С. Милашук² \\ ${ }^{1}$ К. т. н., доцент, доцент кафредры экономики и организации строительства учреждения образования \\ «Брестский государственный технический университет», Брест, Беларусь, e-mail: pmkuzmich@tut.by \\ ${ }_{2}^{2}$ Магистр технических наук, ассистент кафредры экономики и организации строительства учреждения образования \\ «Брестский государственный технический университет», Брест, Беларусь, e-mail: lionejja@mail.ru
}

\begin{abstract}
Рефрерат
Главной задачей календарного планирования является составление таких графиков выполнения работ, которые будут удовлетворять всем необходимым ограничениям, в том числе и по ресурсам, в наибольшей степени обеспечивая равномерность их потребления.

В данной статье приводится анализ существующих методик оценки календарных планов. Рассматриваются положительные и отрицательные стороны показателей, по которым выполняется их оценка. Также предложен новый показатель для комплексной оценки качества календарных планов: коэффициент неравномерности потребления ресурсов.
\end{abstract}

Ключевые слова: коэффициенты потребления ресурсов, планирование, календарный план, качество планирования, график потребности.

\section{EVALUATION OF CALENDAR PLANS USED IN OPERATIONAL AND TACTICAL MANAGEMENT}

Abstract

\section{P. M. Kuzmich, E. S. Milashuk}

The main task of calendar planning is to draw up such work schedules that will meet all the necessary constraints, including the number of resources, and to the greatest extent ensure the uniformity of their consumption.

This article provides an analysis of existing methods for evaluating calendar plans. The positive and negative aspects of the indicators for which they are evaluated are considered. A new indicator is also proposed for a comprehensive assessment of the quality of calendar plans: coefficient of uneven resource consumption.

Keywords: resource consumption coefficients, planning, calendar plan, quality of planning, demand schedule.

\section{Введение}

В управлении проектами, планировании производства работ подрядными организациями по капитальному ремонту, реконструкции, возведению зданий и сооружений применение календарных планов весьма целесообразно по следующим соображениям:

- своевременное и, по возможности, равномерное обеспечение (возобновляемыми) ресурсами, материалами и конструкциями;

- увязка во времени и пространстве работы подрядчиков, субподрядчиков, соисполнителей, исключающая их простои в ожидании фронта работ;

- увязка потребностей в фринансовом обеспечении с возможностями финансирования.

Под календарным планом понимают проектный документ, определяющий последовательность выполнения работ, процессов (реализации процедур), диктуемую требованиями технологического характера, сроки и календарные даты их выполнения.

Календарные планы могут быть представлены в различных формах:

1) графической:

a) сетевые модели в форматах работы-дуги или работы-события;

б) линейная диаграмма Гантта (линейный график);

в) циклограмма.

2) матричной (табличной)

В основе календарного плана лежит организационно-технологическая модель возведения объекта, выполнения комплекса работ по капитальному ремонту, реконструкции, реставрации и т. п. Её составление диктуется технологическими взаимосвязями работ. Далее определяются продолжительности работ и особенности их взаимосвязей, а именно возможности выполнения работ параллельно или последовательно. Если технологически работы должны выполняться последовательно, то возможно ли их частичное совмещение, то есть начало выполнения последующей работы после частичного выполнения предшествующей (отступ). Этот отступ определяется технологическими особенностями предшествующей и последующей работ, но в календарном плане он трансформируется в присвоение связи между работами временного значения, которое может принимать как положительное, так и отрицательное значение; зависеть от типа связи между работами (окончание-начало, начало-начало, окончание-окончание, начало-окончание).

Процедура составления календарного плана заключается в создании и последующем уточнении расписания, в котором учитывается состав работ, ограничения по срокам, ресурсам, сезонные ограничения. Календарное планирование включает несколько стадий: планирование содержания проекта (состав работ, процессов, процедур), построение структуры работ, выстраивание последовательности работ, составление модели (графика), составление плана сроков, продолжительностей, согласование логических связей работ, определение потребности в ресурсах, составление плана использования ресурсов.

Из этого следует, что календарный план представляет собой совокупность документов, планов, графиков и т. п., основными из которых являются:

1) собственно организационно-технологическая модель в одной из вышеперечисленных форм, привязанная к календарной основе;

2) графики (планы) потребности (потребления) в возобновляемых ресурсах;

3) графики (планы) потребности в невозобновляемых ресурсах (энергоресурсы, материалы, конструктивные изделия, оборудование и т. п.). Из всех этих составляющих наибольший интерес представляют графики потребности в возобновляемых ресурсах, к которым следует отнести:

а) графики потребности в рабочих, машинистах, специалистах, работающих

б) графики работы машин и механизмов.

\section{Анализ методик оценки календарных планов}

Существуют различные подходы в оценке качества календарных планов. Для этих целей, как правило, все календарные планы сопровождаются технико-экономическими показателями (ТЭП). В наиболее распространенном варианте в них включают: сметную стоимость работ, комплекса работ, капитального ремонта, реконструкции, возведения объекта; их трудоемкость и машиноемкость; выработку на 1 человеко-день, нормативную и планируемую продолжительность строительства и т. п.

Как видно из этого перечня и из [1], «Практика календарного планирования строительного производства показывает, что все 
Вестник Брестского государственного технического университета. 2021

показатели, используемые при оценке календарных планов выполнения CMP, имеют различную природу и измеряются в различных масштабах». В связи с чем они ограничено пригодны для оценки в том числе количественной, отдельно взятого календарного плана и могут быть использованы лишь при сравнении последнего с планами по аналогичным объектам или типовыми показателями по уже возведенным объектам, причём объекты должны иметь схожие конструктивно-технологические параметры.

Известен также показатель непрерывности ведения работ $K_{H}$ [2], который определяется отношением времени непрерывного выполнения работ на объекте $t_{\phi}$ к числу календарных рабочих дней $t_{\kappa} \mathrm{c}$ учетом перерывов $t_{m}$

$$
K_{H}=\frac{t_{\phi}}{t_{K}-t_{m}} .
$$

Данный показатель предлагается для оценки уровня организации производства, но он может быть использован и для оценки качества календарного плана.

Наиболее распространенным показателем, используемым для оценки качества календарного плана, является коэффициент неравномерности $K$ [3], определяемый соотношением максимальной численности ресурса (рабочих) $N_{\text {max }}$ к его среднему значению (average) $N_{a v}$ :

$$
K=\frac{N_{\max }}{N_{a v}}
$$

Широкое распространение данного способа объясняется его простотой, близкой к примитивности, так как значение $N_{\max }$ для конкретного календарного плана может быть неприемлемо большим, что даст нам неприемлемое значение $K$, но безальтернативным в силу специфики объекта, комплекса работ.

Наиболее объективную оценку качества решения задачи определения оптимальной продолжительности строительства, а следовательно, и качество календарного плана можно получить, если оценить варианты календарных планов по критерию $3_{c}$ (минимум всех затрат) [4], который для наилучшего варианта принимает минимальное значение. $3_{c}$ предлагается определять как сумму:

$$
3_{c}=C^{c}+\Delta \Pi 3+\Delta H P,
$$

где $C^{C}$ - сметная стоимость здания (сооружения);

$\Delta$ ПЗ - дополнительные прямые затраты;

$\Delta H P$ - дополнительные накладные расходы (общехозяйственные и общепроизводственные расходы).

Использование критерия $3_{c}$ для оценки календарных планов сопряжено с большим объёмом расчетов по определению сметной стоимости, прямых затрат, ОХРИОПР для различных вариантов календарных планов. Также при изменении составляющих в сметной стоимости строительства, таких как прямые затраты и ОХР $О П Р$ будет изменяться и прибыль. Критерий $3_{c}$ это не учитывает. Увеличение объемов расчётов приводит к существенному повышению трудоемкости календарного планирования. Кроме этого, на стадии разработки календарного плана не все составляющие прямых затрат, ОХРИОПР могут быть определены в полной мере соответствующими фактическим. Поэтому от критерия $3_{c}$ следует отказаться.

«Сравнение вариантов календарных планов с целью выбора наилучшего из них предполагает, что все они могут быть оценены различными критериями» [1]. Для этого предлагается комплекс показателей:

- стоимость работ $C$;

- время выполнения работ $T$;

- эффективность использования ресурсов во времени $К_{\text {эф }}$

- совмещение разнотипных видов работ $K_{\text {св; }}$;

- непрерывность использование ресурсов $K_{H}$;

- критичность работ $K_{\kappa р}$;

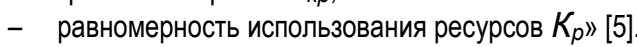

Все перечисленные показатели, за исключением коэффициента равномерности использования ресурсов $K_{p}$, носят частный характер, не дающий объективного, объёмного представления о качестве календарного плана.
Коэффициент равномерности использования ресурсов $K_{p}$ предлагается определять по следующей формуле:

$$
\ll K_{p}=\sum_{i=1}^{K}\left(1-\frac{f_{i}}{F_{i}}\right) \frac{K_{i}}{K},
$$

где $f_{i}$ - суммарная площадь участка, выступающего над линией равномерного потребления ресурсов;

$F_{i}$ - площадь участка, ограниченного линией равномерного потребление ресурсов;

$K_{i}$ - количество $i$-го вида ресурсов;

K - общее количество ресурсов» [5].

Данный подход, на наш взгляд, имеет следующие недостатки:

- не учитывается площадь участков, находящихся ниже линии равномерного потребления ресурсов, то есть их «вклад» в «неравномерность» принимается равным нулю. С этим нельзя согласиться;

- неясно как определить площадь участка, ограниченного линией равномерного потребления ресурсов;

- как, в каких единицах измерять количество ресурсов;

- каково желаемое, идеальное значение может принимать $K_{p}$ чему он должен быть равен в идеале.

Ориентируясь на какое значение $K_{p}$, мы должны оценивать качество календарного плана, к какому значению мы должны стремиться, составляя календарный план?

Оценка календарных планов по коэфффициенту неравномерности в ресурсах

В отличие от стратегического в оперативном и тактическом управлении, учитывая специфику строительного производства, равномерное потребление ресурсов не следует рассматривать как безусловно достижимое даже в безупречно разработанном календарном плане. При поточной организации строительного производства определяющим является специализация исполнителей и, в зависимости от вида работ, их количество может отличаться, и притом существенно. Отсутствие специализации позволяет возводить объекты, выполнять работы исполнителями в неизменном составе, но это сопровождается снижением качества и низкой производительностью. Поэтому стремление к равномерности потребления ресурсов не следует рассматривать как абсолютную цель, а лишь как направление, в котором следует искать наилучшее решение при разработке календарных планов.

Поэтому в общем виде оценку равномерности потребления ресурсов как показателя качества календарного плана следует давать, сопоставляя некий желаемый, но необязательно идеальный, абсолютно равномерный график потребности в них с планируемым, полученным в результате составление календарного плана. С этой целью вместо коэффициента равномерности потребления ресурсов следует ввести коэффициент неравномерности $K_{\text {нер}}$, который в большей мере будет отвечать целям оценки календарных планов, определяемый по следующей формуле (рисунок 1):

$$
K_{\text {нер }}^{i}=\frac{\sum_{i=1}^{n-1}\left|\int_{a_{1}}^{a_{2}} f_{r^{-}} \int_{a_{2}}^{a_{n}} f_{a v}\right|}{\int_{0}^{T} f_{r}},
$$

где $f_{r}$ - функция, описывающая полученный в результате разработки календарного плана график потребления $i$-го ресурса;

$f_{a v}$ - функция, описывающая желаемый идеальный график потребления $i$-го ресурса;

$n$ - количество участков отклонения графиков;

$a_{1}, a_{2}, \ldots, a_{n}-$ ординаты этих участков;

$T$ - общая продолжительность работ (длина календарного плана). Q

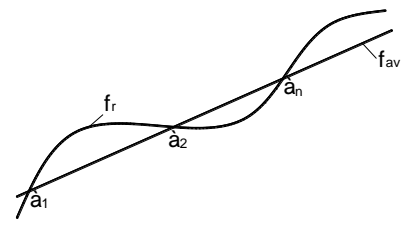

Рисунок 2 - График потребности в ресурсе (в общем виде) 
Следует признать, что в большинстве случаев ресурсы описываются дискретными величинами и описание их в виде непрерывной функции требует значительного объема статистических данных, которые зачастую не представляется возможным получить. Чаще всего графики потребности в ресурсах описываются эпюрами в форме гистограмм. Поэтому для комплексной оценки качества календарных планов, применяемых в оперативном и тактическом управлении, коэффициент неравномерности использования ресурсов предлагается определять как отношение суммы площадей эпюры потребности в ресурсе расположенных как выше среднего (равномерного) значения потребности в ресурсе, так и ниже этой линии (рисунок 2):

$$
K_{H е р}^{i}=\frac{\sum_{j=1}^{m} f_{j}+\sum_{k=1} S_{k}}{N_{a v}^{i} \cdot T},
$$

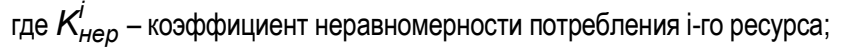

$f_{j}$ - площадь участков, расположенных выше линии среднего (равномерного) значения потребности в ресурсе;

$S_{k}$ - площадь участков, расположенных ниже линии среднего (равномерного) значения потребности в ресурсе;

$T$ - период потребности в ресурсе (нахождения его на объекте);

$N_{a v}^{i}$ - среднее (равномерное) значение потребности в ресурсе:

$$
N_{a v}=\frac{\sum_{i=1}^{n} t_{i} \cdot N_{i}}{T},
$$

где $N_{i}$ - потребность в ресурсе;

$t_{i}-$ временной период.

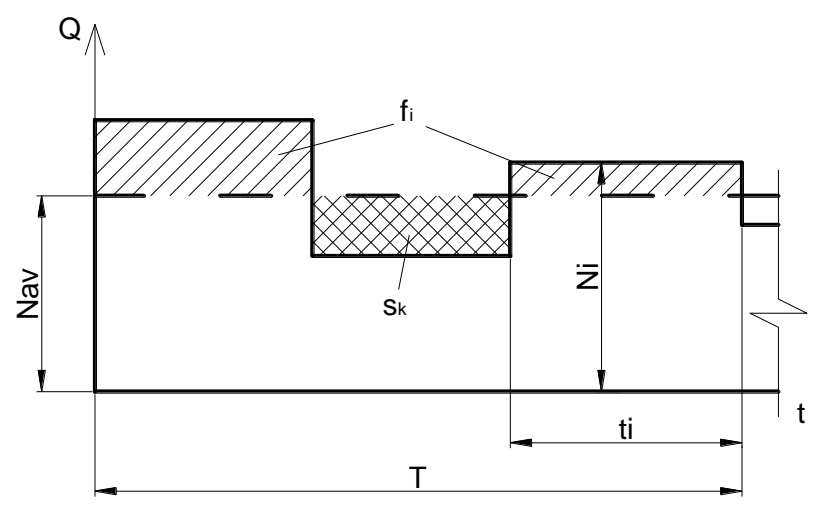

Рисунок 2 - График потребности в ресурсе

Комплексная оценка качества календарного плана по всей совокупности ресурсов может быть оценена коэффрициентом $K_{\text {нер, по }}$ следующей формуле:

$$
K_{\text {Hер }}=\frac{\sum_{i=1}^{n} \kappa_{H е p}^{i}}{n},
$$

где $n$ - количество ресурсов, предусмотренных для реализации проекта, возведение объекта в календарном плане.

Степень привлечения ресурсов, предусмотренных календарным планом, их количество, вклад в реализацию проекта различны.

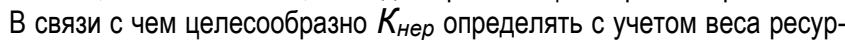
ca. Вес ресурса удобнее и проще всего определять как его сметную стоимость или себестоимость $\left(C_{i}\right)$. Тогда:

$$
K_{\text {нер }}=\frac{\sum_{i=1}^{n} C_{i} \cdot K_{\text {нер }}^{i}}{\sum_{i=1}^{n} C_{i}} .
$$

Очевидно, что идеальным, с точки зрения потребления ресурсов, будет календарный план, коэффициент неравномерности потребления ресурсов которого принимает значение равное нулю (нулевая неравномерность). Очевидно и то, что такой календарный план на объект, комплекс работ составить практически невозможно. Поэтому из нескольких вариантов календарного плана следует

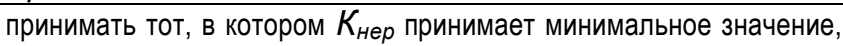
рассматривая его как основной показатель качества календарного плана.

\section{Заключение}

В итоге, для оценки качества календарных планов, на наш взгляд, коэффициент неравномерности потребления ресурсов $K_{\text {нер }}$ может быть использован в качестве основного критерия. Его можно, а в ряде случаев и необходимо, определять по каждому ресурсу отдельно или как комплексный показатель по всем ресурсам.

Кроме коэффициента неравномерности потребления ресурсов, для оценки качества календарных планов следует использовать показатели трудоемкости и машиноемкости. Целесообразно определять и показывать нормативную и планируемую трудоемкости и машиноемкости, а также их удельные значения, определяемые как отношение абсолютных показателей к некоторой существенной характеристике объекта.

И, безусловно, любой календарный план определяет общую продолжительность работ по возведению, реконструкции, капитальному ремонту объекта $T$, которая не может превышать некоторого заданного значения (нормативного, директивного, договорного). При решении задачи по определению $T$ удобнее всего использовать методику по решению обратной задачи календарного планирования [6]. От использования стоимостных показателей для оценки качества календарных планов на стадии оперативного и тактического управления следует отказаться по изложенным выше причинам.

\section{Список цитированных источников}

1. Баркалов, С. А. Управление проектами в строительстве / С. А. Баркалов, В. Ф. Бабкин. - М. : АСВ, 2003. - 288 с.

2. Луканин, Б. Ф. Количественная оценка повышения эффективности организации строительного производства / Б. Ф. Луканин, Ф. Р. Уфимцев, Ю. Д. Шахов. - М. : Стройиздат, 1981. - 124 с.

3. Сухачев, И. А. Организация и панирование строительного производства. Управление строительной организацией / И. А. Сухачев. М. : Стройиздат, 1989. - 752 с.

4. Хибухин, В. П. Математические методы планирования и управления строительством / В. П. Хибухин, В. З. Величкин, В. И. Втюрин. - М. : Стройиздат, 1990. - 184 с.

5. Баркалов, С. А. Разработка и исследование моделей и механизмов оптимизации и оценки календарных планов в управлении проектами : дис. ... докт. техн. наук : 05.13.10 / С. А. Баркалов. М., 1999. - 401 л.

6. Кузьмич, П. М. Прямая и обратная задачи календарного планирования / П. М. Кузьмич, Е. С. Милашук // Вестник Брестского государственного технического университета, 2020. - № 3 : Экономика. - С. 46-48.

\section{References}

1. Barkalov, S. A. Upravlenie proektami v stroitel'stve / S. A. Barkalov, V. F. Babkin. - M. : ACB, 2003. - $288 \mathrm{~s}$.

2. Lukanin, B. F. Kolichestvennaya ocenka povysheniya effektivnosti organizacii stroitel'nogo proizvodstva / B.F. Lukanin, F. R. Ufimcev, Yu. D. SHahov. - M. : Strojiz-dat, 1981. - 124 s.

3. Suhachev, I. A. Organizaciya i panirovanie stroitel'nogo proizvodstva. Upravlenie stroitel'noj organizaciej / I. A. Suhachev. - M. : Strojizdat, 1989. - $752 \mathrm{~s}$.

4. Hibuhin, V. P. Matematicheskie metody planirovaniya i upravleniya stroitel'stvom / V. P. Hibuhin, V. Z. Velichkin, V. I. Vtyurin. - M. : Strojizdat, 1990. - $184 \mathrm{~s}$.

5. Barkalov, S. A. Razrabotka i issledovanie modelej i mekhanizmov optimizacii i ocenki kalendarnyh planov v upravlenii proektami : dis. ... dokt. tekhn. nauk : 05.13.10 / S. A. Barkalov. - M., 1999. - 401 I.

6. Kuz'mich, P. M. Pryamaya i obratnaya zadachi kalendarnogo planirovaniya / P. M. Kuz'mich, E. S. Milashuk // Vestnik Brestskogo gosudarstvennogo tekhniche-skogo universiteta, 2020. - № 3 : Ekonomika. S. 46-48.

Материал поступил в редакцию 05.05.2021 